International Journal of Network Security \& Its Applications (IJNSA), Vol.3, No.2, March 2011

\title{
A QoS Based MAC Protocol For Wireless Ad-hoc Network
}

\author{
Vandana khare \\ Associate professor, ECE \\ CMEC,Hyd(A.P) \\ Email:vandna369@gmail.com
}

\author{
Dr. D. SrinivasRao \\ Professor- ECE \\ JNTUH,Hyd (A.P) \\ Email: dsraoece@yahoo.co.uk
}

\author{
Dr. Y. Madhavee Latha \\ Principal \\ MRECW, Hyd(A.P) \\ Email: madhuvsk2003@yahoo.co.in
}

\begin{abstract}
Multimedia communication over wireless Ad-hoc networks has become the driving technology for many of the important applications, experiencing dramatic market growth and promising revolutionary experiences in personal communication, gaming, entertainment, military, security, environment monitoring, and more. The advances in wireless communications and growth of real-time applications have necessitated the development of wireless networks that can support high Quality of Service $(Q o S)$ and power control. A node in an ad hoc network is normally battery operated which poses a huge constraint on the power consumption of such a node. Hence, designing a power efficient MAC protocol for ad hoc wireless networks is a major challenge. In this paper, we propose a CDMA based power controlled medium access protocol for mobile and ad hoc network (MNA). The protocol conserves power and provides QoS guarantees for multimedia traffics. In that network one of the fundamental challenger in MANETs is how to increase the overall network throughputs well as reading the delay while maintaining how energy consumption for packet processing in communication. simulation results shows that the performance of the protocol with increase in traffic while QoS is better in terms of Energy consumption, throughput, \& communication delay than existing protocol.
\end{abstract}

Keywords: QoS, MANETS, Multimedia, CDMA throughput, delay.

\section{INTRODUCTION}

In recent years wireless communication networks have become increasingly popular. Many types of wireless services have become available, including cellular systems, satellite communication networks, and wireless local area networks (WLANs) [1, 2]. The increasing popularity of WLANs and wireless devices has led to greater interest in wireless ad hoc networks. An ad hoc network [3] is formed by wireless, potentially mobile hosts, without requiring the use of any fixed infrastructure, and can be set up in the environment where the wiring of a conventional network is difficult or not economically feasible. Wireless ad hoc networks face challenges that are not present in wired networks. In wired networks, transmission errors typically occur at a low rate and interference among different communication flows is minimal. Collision detection is usually fast and easy in wired networks. Wireless communication, however, requires a shared transmission medium that is highly error-prone. Hence, in wireless communication, there is a much higher chance for collisions to occur. It is also more difficult to detect a collision in a wireless network. Often the lack of a reply message is the only way for a node to detect a collision. Therefore, compared to a wired network, a wireless network requires a different and more complicated medium access control (MAC) layer. This paper focuses on the issues on MAC layer for wireless networks. The rest of this paper is organized as follows: Section II discusses the basics of video compression and the 802.11 protocol stack respectively. Section III outlines the video QoS enhancing algorithm. Section IV discusses the simulation set up. Section V enumerates and analyses the simulation results. Finally, we conclude the paper in Section VI. 
International Journal of Network Security \& Its Applications (IJNSA), Vol.3, No.2, March 2011

\section{RELATED WORK}

Several CDMA-based MAC protocols for MANETs have been proposed in past. These protocols, in general, are based on random channel access, whereby a terminal with a packet to transmit can proceed immediately with its transmission, irrespective of the state of the channel. We refer to such schemes as random access CDMA (RA-CDMA). Under appropriate code assignment and spreading-code schemes, RA-CDMA protocols are guaranteed to be free of primary collisions. However, the nonzero cross-correlations between different CDMA codes can induce multi-access interference (MAI), resulting in secondary collisions at a receiver (collisions between two or more transmissions that use different CDMA codes). This problem is known as the near-far problem. The near-far problem can cause a significant reduction in network throughput, and is to be overcome for designing CDMA-based MAC protocols for MANETs.

For the integration of CDMA with ad hoc architecture various proposal were made in past. In [4] the addresses part of the packet are spread using the common code, while the rest of the packet is spread using the transmitter-based approach. A receiver notes the address of the source terminal and uses this address to switch to the corresponding code. In [5] the authors proposed the coded tone sense protocol, in which $\mathrm{K}$ busy tones are associated with $\mathrm{K}$ spreading codes. During packet reception on a certain code, the receiving station broadcasts the corresponding busy tone. In [6] all terminals send the RTS-CTS packets on a common code, while the data packets are sent using a transmitter- or a receiver-based approach. Somewhat similar approaches were proposed in [7] and [8]. In all the above protocols, the authors assume perfect orthogonality between spreading codes, i.e., they ignore the near-far problem.

A reservation-based scheme was proposed in [9], whereby small control packets are used to request slot assignments for data packets. The authors investigated the use of FHSS to avoid MAI. Their approach, however, cannot be used for DSSS, which is the method of choice in recent wireless standards (e.g. IS-95). In [10] and [11] the authors proposed distributed channel assignment algorithms for SS Multihop networks. Those protocols, however, do not allow for any MAI, and hence cannot support concurrent transmissions of signals with different codes. Clustering as proposed in [12] is another interesting approach for power control in CDMA networks. It simplifies the forwarding function for most terminals, but at the expense of reducing network utilization (since all communications have to go through the cluster heads). This can also lead to the creation of bottlenecks.

In [13] the authors proposed the use of a multi-user detection circuit at the receiver to mitigate the near-far problem in MANETs. The proposed scheme also requires the use of GPS receivers to provide accurate position and timing information. Such a scheme relies heavily on physical layer techniques to mitigate MAI, and makes no effort to account for MAI at the MAC layer. Moreover, although it is feasible to deploy multi-user GPS receivers at the base station, presently it is impractical (and expensive) to implement such receivers within the mobile terminal. Recently, an interesting approach for joint scheduling and power control in ad hoc networks was proposed [14]. This approach, however, requires a central controller for executing the scheduling algorithm, i.e., it is not a truly distributed solution. Furthermore, it assumes the existence of a separate feedback channel that enables receivers to send their SNR measurements to their respective transmitters in a contention free manner. In [15] and [16] the authors analyzed RA-CDMA protocols for MANETs in the presence of MAI. They assumed that transmissions of all neighbors produce the same noise effect, and therefore, the SNR 
International Journal of Network Security \& Its Applications (IJNSA), Vol.3, No.2, March 2011

threshold can be converted into a threshold on the number of transmissions (n) in the receiver's neighborhood. A packet is correctly received when that number is less than the predetermined threshold $\mathrm{n}$. hence; the protocol was called CDMA/n. Although such an approximation may not be accurate in topologies where nodes are not equally spaced, it shows that MAI can significantly degrade network performance in such approaches

\section{PROPOSED PROTOCOL CONCEPT}

The users are the sources which send the message. This message will be coded and the signal will be modulated by the carrier signal. The codes will be generated with the linear feedback shift register (LFSR) mechanism. The generated code is known as pseudo random noise sequence. For example when the message is of 8 bit length, The generated code will be of 32 bit length. This code is generated for single user. So similarly we can generate different codes for different users and modulate these signals by using modulator. And these signals are transmitted by transmitting antenna. The generation of the code plays an important role in the CDMA communication system. For generating these codes we use the LFSR mechanism. The band width present for the user can be utilized properly in CDMA communication system. As the CDMA communication system sends the data for ' $n$ ' number of users at a time. So we can transmit the number of data of different users at the same time. All the messages coded will be kept in the transmitting section to transmit the data. So the transmitted data contains number of messages produced by the different sources. The receiving antenna receives the signals and demodulates the signal in the block of demodulator. The message received will be decoded after demodulation. The decoded message is send to the matched filter. The matched filter matches the data and sends to de-spreader to make the message to reach destination. Hence the messages generated by the different users will be transmitted and will be received by the different users at different places which makes the global requirement comes true by using CDMA communication system. The source generates the message. The CDMA code is allocated to the message generated by the source. The network creation takes place for the generated code by the CDMA communication system. The source/destination routes the message in the desired manner. Finally selects an optimal path for transferring the message. Request to send (RTS) and clear to send (CTS) mechanisms are verified and the message is send i.e. performance of the controlled mechanism is performed using the RTS and CTS mechanisms.

\section{DESIGN APPROACH}

Several CDMA-based MAC protocols for MANETs have been proposed in the literature $[1,2,3,4]$. These protocols, in general, are based on random channel access, whereby a terminal with a packet to transmit can proceed immediately with its transmission (starting, possibly, with an RTS/CTS exchange), irrespective of the state of the channel. We refer to such schemes as random access CDMA (RA-CDMA). Under appropriate code assignment and spreading-code schemes, RA-CDMA protocols are guaranteed to be free of primary collisions. However, as explained the nonzero cross-correlations between different CDMA codes can induce multi-access interference (MAI), resulting in secondary collisions at a receiver (collisions between two or more transmissions that use different CDMA codes). In the literature, this problem is known as the near-far problem. As shown in Section 2, the near-far problem can cause a significant reduction in network throughput, and hence cannot be overlooked when designing CDMA-based MAC protocols for MANETs. Accordingly, the 
International Journal of Network Security \& Its Applications (IJNSA), Vol.3, No.2, March 2011

main goal of this paper is to provide a CDMA-based MAC solution for MANETs that addresses the near-far problem.

In our protocol, the transmission powers are dynamically adjusted such that the MAI at any receiver is not strong enough to cause a secondary collision. This results in a significant improvement in network throughput at no additional cost in energy consumption. In fact, the proposed protocol is shown to achieve some energy saving compared to the 802.11 schemes. To the best of our knowledge, this is the first attempt to address the near-far problem in the design of MAC protocols for MANETs.

\subsection{THE NEAR-FAR PROBLEM IN RA-CDMA}

The roots of the near-far problem lies in the fact that unlike FDMA and TDMA channels which can be completely orthogonal, CDMA codes suffer from nonzero crosscorrelation between codes. When a CDMA receiver de-spreads a signal, it effectively computes the cross-correlation between the signal and a locally generated PN sequence. If this PN sequence is identical to the one used to spread the signal at the transmitter (i.e., the message is intended to this receiver), cross-correlation computations restore the original information data. Otherwise, such computations result in either a zero or a nonzero value, depending on whether the system is synchronous or asychnronous. A system is called time-synchronous if all signals originate from the same transmitter, as in the case of the downlink of a cellular CDMA network. In here, synchrony is manifested in two ways. First, different transmissions that are intended for different receivers will have a common time reference.

Second, from the viewpoint of a given mobile terminal, all signals (intended or not) propagate through the same paths, and thus suffer the same time delays. In synchronous systems, it is possible to design completely orthogonal spreading codes. In fact, in the IS-95 standard for cellular CDMA networks [19], each user of the channel is assigned a Hadamard (or Walsh) code. These codes are orthogonal and are used to "channelize" the available bandwidth.

On the other hand, a system is called time-asynchronous if signals originate from multiple transmitters, as in the case of the uplink of cellular networks and also in MANETs. The reasons behind the naming are twofold. First, since signals originate from different transmitters, it is generally not feasible to have a common time reference for all the transmissions that arrive at a receiver. Second, these transmissions propagate through different paths; thus, they suffer different time delays [20]. In an asynchronous system, it is not possible to design spreading codes that are orthogonal for all time offsets [19]. In this case, the crosscorrelation between codes cannot be neglected. In fact, codes that is orthogonal in synchronous systems (e.g., Hadamard codes) exhibit high cross-correlation when not perfectly synchronized. Instead, PN codes that are designed specifically to have low cross-correlation are used. While the code design problem is crucial in determining the system performance, of greater importance is the problem of nonzero cross-correlation of the PN codes [21]. Unintended transmissions add nonzero MAI during the dispreading at a receiver. The near-far problem is a severe consequence of MAI, whereby a receiver who is trying to detect the signal of the ith transmitter may be much closer in distance to, say, the $\mathrm{j}^{\text {th }}$ transmitter than the ith transmitter. When all transmission powers are equal, the signal from the $\mathrm{j}^{\text {th }}$ transmitter will arrive at the receiver in question with a sufficiently larger power than that of the $i^{\text {th }}$ transmitter, causing incorrect decoding of the $\mathrm{i}^{\text {th }}$ transmission (i.e., a secondary collision). 
International Journal of Network Security \& Its Applications (IJNSA), Vol.3, No.2, March 2011

\subsection{ALLOCATION SCHEME}

In our design, we use two frequency channels, one for data and one for control (i.e., FDMA-like partitioning). A common spreading code is used by all nodes over the control channel, while several terminal-specific codes can be used over the data channel. This architecture is shown in Figure 4.1. Note that the different codes used over the data channel are not perfectly orthogonal. However, because of the frequency separation, a signal over the control channel is completely orthogonal to any signal (or code) over the data channel. The splitting of the available bandwidth into two non-overlapping frequency bands is fundamentally needed to allow a terminal to transmit and receive simultaneously over the control and data channels, irrespective of the signal power. As explained, this protocol utilizes the fact to allow interference-limited transmissions that use (quasiorthogonal) data channel codes to proceed concurrently.

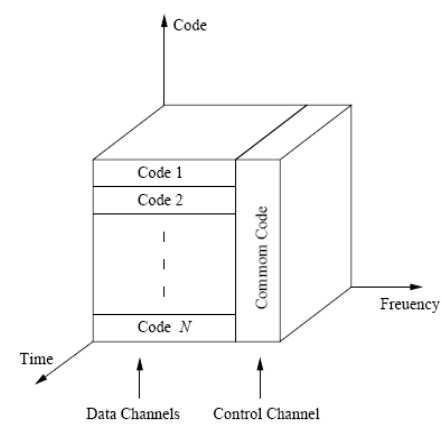

Figure 4.1: Data and control codes in the proposed protocol.

\subsection{Channel Model}

In designing the protocol,

(1) The channel gain is stationary for the duration of the control and the ensuing data packet transmission periods;

(2) The gain between two terminals is the same in both directions.

(3) Data and control packets between a pair of terminals observe similar channel gains.

\subsubsection{NOISE IN COMMUNICATION SYSTEMS:}

Noise plays a crucial role in communication systems. In theory, it determines the theoretical capacity of the channel. In practice it determines the number of errors occurring in a digital communication.

Noise is a random signal whose value cannot be predicted. It can only be assumed of taking a particular value, or range of values randomly. The probability density function (pdf) $p(\mathbf{x})$ of a random signal, or random variable $x$, is defined to be the probability that the random variable $x$ takes a value between $\boldsymbol{\Sigma}_{0}$ and $\boldsymbol{w}_{\boldsymbol{0}}$. Defined as:

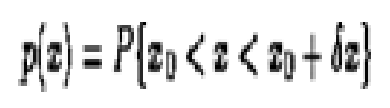


International Journal of Network Security \& Its Applications (IJNSA), Vol.3, No.2, March 2011

The probability that the random variable will take a value lying between ${ }^{\boldsymbol{F}_{\mathbf{1}}}$ and ${ }^{\boldsymbol{E}_{\boldsymbol{x}}}$ is then the integral of the pdf over the interval $\mathbf{x}_{\mathbf{2}}-\mathbf{2} \mathbf{1}$ :

The probability $P\{-\infty<a<\infty\}$ is unity. Thus:

$$
P\left\{x_{1}<x<x_{2}\right\}=\int_{x_{1}}^{x_{2}} p(x) d x
$$$$
\int_{-\infty}^{\infty} p(x) d x=1
$$

A density satisfying equation is termed normalised.

The probability distribution function $P(\boldsymbol{s})$ is defined to be the probability that a random variable, $\mathbf{x}$ is less than $\mathbf{E}$ :

$$
P\left(x_{0}\right)=P\left\{x<x_{0}\right\}=\int_{-\infty}^{x_{0}} p(x) d x
$$

From the rules of integration:

$$
\begin{gathered}
P\left\{x_{1}<x<x_{y}\right\}=P\left(z_{y}\right)-P\left(x_{1}\right) \\
P(\infty)=1, \quad P(-\infty)=0
\end{gathered}
$$

The meaning of the two functions $\boldsymbol{P ( \boldsymbol { x } )}$ and $\boldsymbol{P ( \boldsymbol { x } )}$ become clearer with the aid of examples.

Continuous distribution. An example of a continuous distribution is the Normal, or Gaussian distribution:

$$
p(x)=\frac{1}{\sigma \sqrt{2 \pi}} c c^{\frac{-(x-n)^{2}}{2 \sigma^{2}}}
$$

where $m$ is the mean value of ${ }^{p(\boldsymbol{x})}$. The constant term ensures that the distribution is normalised. This expression is important as many naturally occurring noise sources can be described by it, e.g. white noise. Further, we can simplify the expression by considering the source to be a zero mean random variable, i.e. $\mathrm{m}=0$.

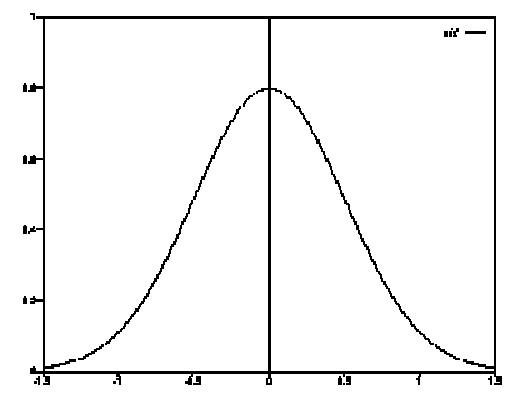

Figure 4.2 A zero mean Gaussian distribution with $\sigma=0.2$ 
How would this be used If we want to know the probability of, say, the noise signal, having the value ${ }^{\mathbf{\pm} \mathbf{u}_{\mathbf{1}}}$, we would evaluate:

$$
P\left[+v_{1}<x<-v_{1}\right]=\int_{x=-v_{1}}^{x=+v_{1}} p(x) d x
$$

In general, to evaluate $P\left(+\boldsymbol{\pi}_{1}<\boldsymbol{x}<-\boldsymbol{\pi}_{\mathbf{1}}\right)$, if we use:

$$
\begin{aligned}
u & =\frac{x}{\sigma \sqrt{2}} \\
d x & =d u \sigma \sqrt{2}
\end{aligned}
$$

then from with $\mathrm{m}=0$ we have:

The distribution function $\boldsymbol{P ( \boldsymbol { x } )}$ is usually written in terms of the error function ${ }^{\mathbf{c r t}(\boldsymbol{x})}$ :

$$
\begin{aligned}
P\left\{+x_{1}<x<-x_{1}\right\} & =\frac{1}{\sqrt{\pi}} \int_{-x_{1}}^{+x_{1}} \mathrm{c}^{-u^{2}} d u \\
& =\frac{2}{\sqrt{\pi}} \int_{0}^{x_{1}} \mathrm{c}^{-u^{2} d u}
\end{aligned}
$$

$$
P(x)=\operatorname{crf}(x)
$$

where $\stackrel{\boldsymbol{c r f}(\boldsymbol{a})}{\text { is given by: }}$

$$
\operatorname{crf}(\boldsymbol{x})=\frac{2}{\sqrt{\pi}} \int_{0}^{x} c^{-u^{2}} d u
$$

\begin{tabular}{|c|c|c|c|}
\hline & $P(x)$ & $\mathbf{x}$ & $P(x)$ \\
\hline 0.5 & 0.5205 & 2.0 & 0.0053 \\
\hline 1, & 0.8427 & 2.5 & 0.0005 \\
\hline & 0.9661 & 3.0 & 0.99897 \\
\hline
\end{tabular}

which has the property:

$$
\operatorname{crf}(x)=-\operatorname{crf}(-x)
$$

The integral is difficult to evaluate and is approximated by use of tables which exist for various

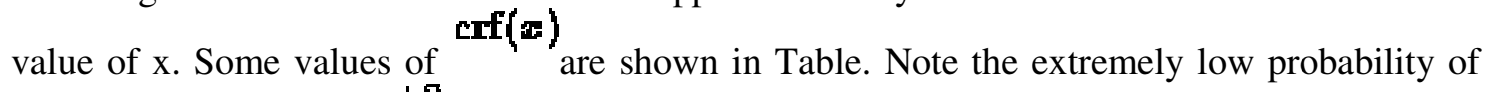
the value falling outside ${ }^{ \pm 2}$.

Table 2: Some values for the error function $P(\boldsymbol{x})$ A more practically useful measure is to consider $\boldsymbol{P}\{-\boldsymbol{k} \sigma<\boldsymbol{x}<+\boldsymbol{k} \boldsymbol{\sigma}\}$, which gives us: 
International Journal of Network Security \& Its Applications (IJNSA), Vol.3, No.2, March 2011

$$
P_{k}(k \sigma)=\operatorname{crf}\left(\frac{k}{\sqrt{2}}\right)
$$

This is useful in that it gives us a feeling of how likely it is that the value of the noise signal will exceed certain thresholds as given by k. A similar table to that of Table can be evaluated for ${ }_{\boldsymbol{k}}$ and is given in Table.

\begin{tabular}{|l|l||l|l|}
\hline $\mathbf{h}$ & $P_{\mathrm{h}}$ & $\mathbf{k}$ & $\boldsymbol{P}_{\mathrm{h}}$ \\
\hline $\mathbf{0 . 5}$ & 0.989 & 2.5 & 0.988 \\
1.0 & 0.689 & 3.0 & 0.907 \\
1.5 & 0.866 & 3.5 & 0.9095 \\
$\mathbf{2 . 0}$ & .955 & $\mathbf{4 . 0}$ & 0.99084 \\
\hline
\end{tabular}

Table1: Some values for the error function,

So we see that it is highly unlikely for our noise signal to take values outside the range $\mathbf{E y \boldsymbol { r }}^{\text {. }}$ The complementary error function, ${ }^{\mathbf{a r f c}(\boldsymbol{\omega})}$, is defined as:

$$
\operatorname{crfc}(x)=1-\operatorname{crf}(x)=\frac{2}{\sqrt{\pi}} \int_{x}^{\infty} c^{-u^{x}} d u
$$

Discrete distribution. Probability density functions need not be continuous. If a random variable can only take discrete values, its pdf takes the forms of lines. An example of a discrete distribution is the Poisson distribution:

$$
p(n)=P\{x=n\}=\frac{\alpha^{n}}{n !} c^{-\alpha}
$$

where $\mathbf{n}=0, \mathbf{1}_{1}, \mathbf{y}_{1} \cdots$. The most celebrated example of the Poisson distribution is its accurate description of the number of men in the Austro-Hungarian army killed by kicks from a horse in each year. We cannot predict the value a random variable may take on a particular occasion. We can introduce measures that summarise what we expect to happen on average. The two most important measures are the mean (or expectation) and the standard deviation.

The mean of a random variable $\mathrm{x}$ is defined to be:

$$
\eta=\int_{-\infty}^{\infty} x p(x) d x
$$

or, for a discrete distribution:

$$
\eta=\sum_{n=-\infty}^{n=\infty} n p(n)
$$

(If $\mathbf{n}$ can take only a limited range of values we adopt the convention that $\boldsymbol{p ( n )}=0$ outside this range).

In the examples above we have assumed that the mean of the Gaussian distribution to be 0 , the mean of the Poisson distribution to is found to be $\boldsymbol{x}$. The mean of a distribution is, in common parlance, the average value. On average 
International Journal of Network Security \& Its Applications (IJNSA), Vol.3, No.2, March 2011

then, the Austro-Hungarian army lost amen per year as a result from horse kicks. The standard deviation is a measure of the spread of the probability distribution around the mean. A small standard deviation means the distribution (and hence occurrences) are close to the mean. A large value indicates a wide range of possible outcomes. The standard deviation $\sigma$ is defined to be:

$$
\sigma^{2}=\int_{-\infty}^{\infty}(x-\eta)^{2} p(x) d x
$$

or, for a discrete distribution:

$$
\sigma^{z}=\sum_{n=-\infty}^{n=\infty}(n-\eta)^{2} p(n)
$$

The square of the standard deviation is called the variance. The Gaussian distribution contains the standard deviation within its definition. The Poisson distribution has a standard deviation of $a^{2}$.

In many cases the noise present in communication signals can be modeled as a zero-mean, Gaussian random variable. This means that its amplitude at a particular time has a pdf. The statement that noise is zero-mean says that, on average, the noise signal takes the value zero. The mean power in the noise signal is equal to the variance of the pdf. We have already seen that the signal-to-noise ratio (SNR) is an important quantity in determining the performance of a communication channel. The noise power referred to in the definition of SNR is the mean noise power. It can therefore be rewritten as:

$\mathrm{SNR}=10 \log \mathrm{s} /\left(\sigma^{*} \sigma\right)$

Moreover, if the source of this noise is thermal noise we that $\boldsymbol{\sigma}^{\mathbf{z}}=\boldsymbol{k} \boldsymbol{T} \boldsymbol{B}$. Irrespective of the source of noise, the variance is sometimes written in the terms of ${ }^{\boldsymbol{m}_{\mathrm{m}}}$ the power per unit $\mathrm{Hz}$, so that:

$$
\sigma^{2}=E_{n} B \text { Watts }
$$

Note that $\boldsymbol{\omega}_{\boldsymbol{n}}^{\boldsymbol{m}_{\text {is }}}$ an energy, because

$$
\text { Encrgy }=\text { Power } \times \text { Time }=\text { Powcr } / \text { Frequency }
$$

\subsection{CONTROLLED ACCESS CDMA (CA-CDMA) PROTOCOL}

CA-CDMA protocol is contention based and uses a modified RTS-CTS reservation mechanism. RTS and CTS packets are transmitted over the control channel (on the common code) at a fixed (maximum) power Pmax. These packets are received by all potentially interfering nodes, as in the IEEE 802.11 scheme.

For the ensuing data packet, the receiver and the transmitter must agree on two parameters: the spreading code and the transmission power. Code selection can be done according to any code assignment scheme. As the transmission power increases, the bit error rate at the intended receiver decreases (i.e., link quality improves), but the MAI added to other ongoing receptions increases. In a CA-CDMA protocol, terminals exploit knowledge of the 
International Journal of Network Security \& Its Applications (IJNSA), Vol.3, No.2, March 2011

power levels of the overheard RTS and CTS messages to determine the power that they can use without disturbing the ongoing receptions.

\subsection{INTERFERENCE MARGIN}

An interference margin is needed to allow terminals at some distance from a receiver to start new transmissions in the future. This margin computation is explained below. Consider an arbitrary receiver i. Let $\mu^{*}$ be the Eb/N0 eff ratio that is needed to achieve the target bit error rate at that receiver. It follows from that to achieve the target error rate, we must have

$$
\frac{P_{0}^{(i)}}{P_{\text {thermal }} \mid P_{\text {MAI }}^{(i)}} \geq \mu^{*}
$$

where $\mathrm{P}_{\text {thermal }}$ is the thermal noise power and $\mathrm{P}(\mathrm{i})_{\text {MAI }}$ is the total MAI at receiver I, so the minimum required received power is $(\mathrm{P}(\mathrm{i}) 0) \min =\mu *(\mathrm{Pthermal}+\mathrm{P}(\mathrm{i}) * \mathrm{MAI})$. The interference margin strongly depends on the network load, which itself can be conveyed in terms of the socalled noise rise $(\xi(i))$, defined as follows:

$$
\xi^{(i)} \stackrel{\text { def }}{=} \frac{\left(\frac{E_{b}}{N_{0}}\right)_{\text {unloaded }}}{\left(\frac{E_{b}}{N_{0}}\right)_{\text {loaded }}}=\frac{P_{\text {thermal }}+P_{\text {MAI }}^{(i)}}{P_{\text {thermal }}}
$$

Note that $(\mathrm{P}(\mathrm{i}) \quad 0$ ) $\mathrm{min}=\xi(\mathrm{i}) \mu *$ Pthermal is also dependent on the noise rise. While more capacity can be achieved by increasing the noise rise (i.e., allowing larger P(i) MAI), the maximum allowable noise rise is constrained by two factors. First, Federal Communications Commission (FCC) regulations limit the power to some fixed value (e.g., 1 Watt for 802.11 devices). Given this maximum transmission power, as the noise rise is increased, the received power (P(i) 0 )min must increase ( $\mu *$ and Pthermal are constants) and hence, the maximum range (or coverage) for reliable communication will decrease. Second, increasing the noise rise increases the power used to transmit the packet, which in turn increases energy consumption. Energy is a scarce resource in MANETs, so it is undesirable to trade off energy for throughput. We set the interference margin used by a transmitter to the maximum planned noise rise

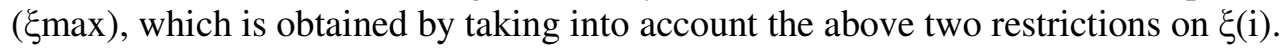

\subsection{ACESSING SCHEME}

The admission scheme allows only transmissions that cause neither primary nor secondary collisions to proceed concurrently. RTS and CTS packets are used to provide three functions. The format of the RTS packet is similar to that of the IEEE 802.11, except for an additional two-byte field that contains the P(j) map value. The format of the RTS packet is as shown in figure 4.2.

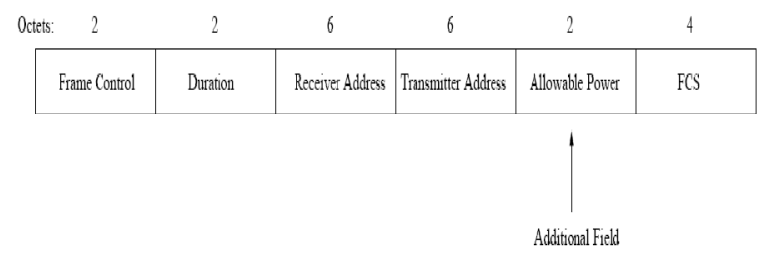

Figure4.3: Format ofthe RTS packet in the CA- CDMA protocol. 
These packets allow nodes to estimate the channel gains between transmitter-receiver pairs. Second, a receiver i uses the CTS packet to notify its neighbors of the additional noise power (denoted by $\mathrm{P}(\mathrm{i})$ noise) that each of the neighbors can add to terminal $\mathrm{i}$ without impacting i's current reception. These neighbors constitute the set of potentially interfering terminals. Finally, each terminal keeps listening to the control channel regardless of the signal destination in order to keep track of the average number of active users in their neighborhoods.

\subsubsection{PACKET TRANSFER:}

The process of packet transfer over the network is explained as follow. If a terminal $\mathrm{j}$ has a packet to transmit, it sends a RTS packet over the control channel at Pmax, and includes in this packet the maximum allowable power level $(P(j)$ map) that terminal $j$ can use that will not disturb any ongoing reception in j's neighborhood. Upon receiving the RTS packet, the intended receiver, say terminal $i$, uses the predetermined Pmax value and the power of the received signal $P(j i)$ received to estimate the channel gain $G_{j i}=P(j i)$ received/Pmax between terminals $i$ and $j$ at that time. Terminal $i$ will be able to correctly decode the data packet if transmitted at a power $\mathrm{P}(\mathrm{ji})$ min given by:

$$
P_{\min }^{(j i)}=\frac{\mu^{*}\left(P_{\text {thermal }}+P_{\text {MAI-current }}^{(i)}\right)}{G_{j i}}
$$

where P(i) MAI-current is the effective current MAI from all already ongoing transmissions. Note that because of the assumed stationarity in the channel gain over small time intervals, Gji is approximately constant throughout the transmissions of the control packet and the ensuing data packet. Now, $\mathrm{P}(\mathrm{ji}) \mathrm{min}$ is the minimum power that terminal $\mathrm{j}$ must use for data transmission in order for terminal $\mathrm{i}$ to correctly decode the data packet at the current level of interference. This $\mathrm{P}(\mathrm{ji}) \mathrm{min}$, however, does not allow for any interference tolerance at terminal $\mathrm{i}$, and thus all neighbors of terminal $\mathrm{i}$ will have to defer their transmissions during terminal i's ongoing reception (i.e., no simultaneous transmissions can take place in the neighborhood of i). The power that terminal $\mathrm{j}$ is allowed to use to send to $\mathrm{i}$ is given by:

$$
P_{\text {allowed }}^{(j i)}=\frac{\xi_{\max } \mu^{*} P_{\text {thermal }}}{G_{j i}}
$$

If $\mathrm{P}(\mathrm{ji})$ allowed $<\mathrm{P}(\mathrm{ji})$ min, then the MAI in the vicinity of terminal $\mathrm{i}$ is greater than the one allowed by the link budget. In this case, $i$ responds with a negative CTS, informing $j$ that it cannot proceed with its transmission. This is to prevent transmissions from taking place over links that provides high MAI. This consequently increases the number of active links in the network (subject to the available power constraints). On the other hand, if $\mathrm{P}(\mathrm{ji})$ allowed $>\mathrm{P}(\mathrm{ji})$ min, then it is possible for terminal i to receive $\mathrm{j}$ 's signal but only if $\mathrm{P}(\mathrm{ji})$ allowed is less than $P(j)$ map (included in the RTS). This last condition is necessary so that transmitter $j$ does not disturb any of the ongoing transmissions in its vicinity. In this case, terminal I calculates the interference power tolerance P(i) MAI-future that it can endure from future unintended transmitters. This power is given by

$$
P_{\text {MAI-future }}^{(i)}=\frac{3 W G_{j i}}{2 \mu^{*}}\left(P_{\text {allowed }}^{(j i)}-P_{\text {min }}^{(j i)}\right)
$$


International Journal of Network Security \& Its Applications (IJNSA), Vol.3, No.2, March 2011

Note that the factor $3 \mathrm{~W} / 2$ comes from the spreading gain. The next step is to equitably distribute this power tolerance among future potentially interfering users in the vicinity of

i. The rational behind this distribution is to prevent one neighbor from consuming the entire $\mathrm{P}(\mathrm{i})$ MAI-future.

The distribution of this power tolerance is given as;

If terminal i keeps track of the number of simultaneous transmissions in its neighborhood, donated by K(i) inst. Monitored by the RTS/CTS exchanges over the control channel. In addition, $\mathrm{i}$ keeps an average $\mathrm{K}(\mathrm{i})$ avg of $\mathrm{K}(\mathrm{i})$ inst over a specified window. Then, $\mathrm{K}(\mathrm{i})$ is calculated as:

$$
K^{(i)}= \begin{cases}\beta\left(K_{\text {avg }}^{(i)}-K_{\text {inst }}^{(i)}\right), & \begin{array}{l}
\text { if } K_{\text {avg }}^{(i)}>K_{\text {inst }}^{(i)} \\
\text { otherwise }
\end{array}\end{cases}
$$

where $\beta>1$ is a safety margin.

While communication it is observed that when the within interference is more than the neighbor interference the level of effect observed is high to reduce this interference effect the neighbor interference is to be reduced. On the calculation if the average interference level per node the CTS packets are generated with the available interference margin with the required power transmission request to the neighboring node as shown in figure 4.3.

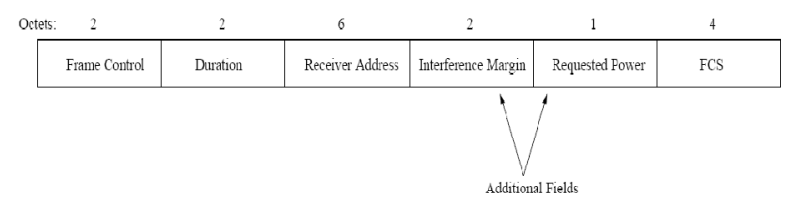

Figure 4.4: Format of the CTS packet in the proposed protocol.

This demanded power Derived from the CTS packet is then compared with the available power limit and transmitted back for acceptance over the control channel to forward the packet. In case the requested power is more than the limiting power the request is denied.

\section{RESULT}

Our scheme is tested for different QoS parameters like throughput parameters, energy consumption, Simultaneous transmission probability, Communication delay.

\subsection{Throughput plot-}

The throughput of Ca-CDMA is less compare to that of the 802.11 with respect to the offered load.

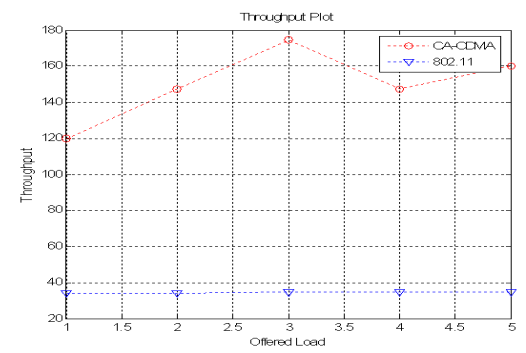

Fig 5.1: Throughput for CDMA Scheme 
International Journal of Network Security \& Its Applications (IJNSA), Vol.3, No.2, March 2011

\subsection{Energy Consumption plot-}

The energy consumption of Ca-CDMA is less compare to that of the 802.11 with respect to the offered load.

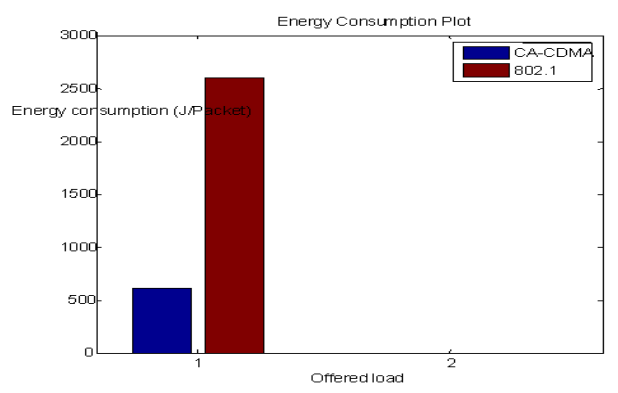

Fig 5.2 Energy consumption for CDMA Scheme

\subsection{Simultaneous transmission probability-}

Simultaneous transmission probability is more in Ca-CDMA communication compared to 802.11 with respect to the load offered.

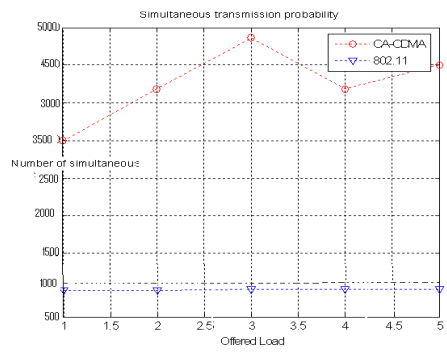

Fig5.3 transmission probability for CDMA Scheme

\subsection{Communication Delay -}

From the above graph we observed that as offered load increases for CA-CDMA with time, communication delay goes down as compared with 802.11.

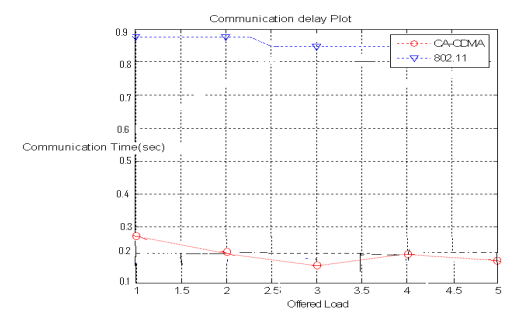

Fig5.4 communication delay for CDMA Scheme

\section{CONCLUSION}

The MAC protocol literature was surveyed to understand the state of the field and the existing level of research. There are several QoS issues in the MAC layer of Ad hoc networks, and the existing MAC protocols have largely concentrated on improving and optimizing these issues in isolation of each other. Here, we introduce a new QoS-aware Mac protocol for Ad hoc networks that simultaneously addresses several QoS issues. In this work, we proposed a 
International Journal of Network Security \& Its Applications (IJNSA), Vol.3, No.2, March 2011

CDMA-based power controlled MAC protocol for wireless ad hoc networks. This protocol, called CA-CDMA, accounts for the multiple access interference, thereby solving the near-far problem that undermines the throughput performance in MANETs. CACDMA uses channelgain information obtained from overheard RTS and CTS packets over an out-of-band control channel to dynamically bind the transmission power of mobile terminals in the vicinity of a receiver. It adjusts the required transmission power for data packets to allow for interferencelimited simultaneous transmissions to take place in the neighborhood of a receiving terminal. We compared the performance of our protocol with that of the IEEE 802.11 scheme. Our simulation results showed that CA-CDMA can improve the network throughput by up to $280 \%$ and, at the same time, achieve 50\% reduction in the energy consumed to successfully deliver a packet from the source to the destination. To the best of our knowledge, CA-CDMA is the first protocol to provide a solution to the near-far problem in CDMA ad hoc systems at the protocol level.

Our future work will focus on other capacity optimizations such as the use of directional antennas in CDMA-based protocols. Because of MAI effects, CDMA benefits significantly from smart antennas. The 802.11 scheme allows nodes to increase their information rate up to $11 \mathrm{Mbps}$ when the power at the receiver is far more than necessary to achieve $2 \mathrm{Mbps}$. It could be possible to improve the proposed scheme by increasing the information rate (i.e. decreasing the processing gain) when the MAI is much less than the planned interference margin when load is low. This is desirable to allow the developed protocol to adapt to different working conditions in terms of the load offered by the users in the network.

\section{REFERENCES:}

[1] S. Chakrabarti and A Mishra, "QoS Issues in Ad Hoc WirelessNetworks", IEEE Communications Magazine, Vol. 39, No. 2, February2001.

[2] J. J. Garcia-Luna-Aceves and J. Raju, "Distributed Assignment of Codes for Multihop Packet-Radio Networks", IEEE MILCOM,November 1997 Barbara Hughes and Vinny Cahill, "Towards Real-time Event-based Communication in Mobile Ad Hoc WirelessNetworks." 2nd International Workshop on real-time LANs in the Internet Age, 2003.

[3] Y. B. Ko and N. H. Vaidya, "Location Aided Routing (LAR) in Mobile Ad Hoc Networks", ACM MOBICOM, 1998.

[4] E. Sousa and J. A. Silvester. Spreading code protocols for distributed spread-spectrum packet radio networks. IEEE Transactions on Communications, 36(3):272-281, Mar. 1988.

[5] K.-W. Hung and T.-S. Yum. The coded tone sense protocol for multihop spread-spectrum packet radio networks. In Proceedings of the IEEE GLOBECOM Conference, volume 2, pages 712-716, 1989.

[6] J. Garcia-Luna-Aceves and J. Raju. Distributed assignment of codes for multihop packetradio networks. In Proceedings of the IEEE MILCOM Conference, volume 1, pages 450-454, 1997.

[7] M. Joa-Ng and I.-T. Lu. Spread spectrum medium access protocol with collision avoidance in mobile ad-hoc wireless network. In Proceedings of the IEEE INFOCOM Conference, volume 2, pages 776-783, 1999. 
International Journal of Network Security \& Its Applications (IJNSA), Vol.3, No.2, March 2011

[8] S.-L. Wu, C.-Y. Lin, Y.-C. Tseng, and J.-P. Sheu. A new multi-channel mac protocol with on-demand channel assignment for multi-hop mobile ad hoc networks. In Proceedings of the International Symposium on Parallel Architectures, Algorithms and Networks, pages 232-237, 2000.

[9] J. E. Wieselthier, A. Ephremides, and J. A. B. Tarr. A distributed reservation-based CDMA protocol that does not require feedback information. IEEE Transactions on Communications, 36(8):913-923, Augut 1988.

[10] I. Cidon and M. Sidi. Distributed assignment algorithms for multihop packet radio networks. IEEE Transactions on Computers, 38(10):1353-1361, Oct. 1989.

[11] A. Ephremides and T. V.Truong. Scheduling broadcasts in multihop radio networks. IEEE Transactions on Communications, 38(4):456-460, Apr. 1990.

[12] T. J. Kwon and M. Gerla. Clustering with power control. In Proceedings of the IEEE MILCOM Conference, volume 2, pages 1424-1428, 1999.

[13] V. Rodoplu and T. Meng. Position based CDMA with multiuser detection (PCDMA/MUD) for wireless ad hoc networks. In IEEE Sixth International Symposium on Spread Spectrum Techniques and Applications, volume 1, pages 336-340, 2000.

[14] T. ElBatt and A. Ephremides. Joint scheduling and power control for wireless ad-hoc networks. In Proceedings of the IEEE INFOCOM Conference, volume 2, pages 976-984, 2002.

[15] M.-S. Chen and R. Boorstyn. Throughput analysis of code division multiple access (CDMA) multihop packet radio networks in the presence of noise. In Proceedings of the IEEE INFOCOM Conference, pages 310-316, 1985.

[16] O. deSouza, M.-S. Chen, and R. R. Boorstyn. A comparison of the performance of protocols in packet radio networks. In Proceedings of the IEEE MILCOM Conference, volume 2, pages $455-460$, Oct 85 . 